$1 \quad$ Supporting information for

\title{
2 Rational Building of Nonblinking Carbon Dots 3 via Charged State Recovery
}

4 Zhihong Wei ${ }^{1} \dagger$, Boyang Wang ${ }^{2} \dagger$, Daocheng Hong $^{3}$, Mingcai Xie $^{1}$, Sushu Wan ${ }^{1}$,

$5 \quad$ Weiqing Yang ${ }^{1}$, Siyu $\mathrm{Lu}^{2 *}$, and Yuxi Tian ${ }^{1 *}$

$6{ }^{1}$ Key Laboratory of Mesoscopic Chemistry of MOE, School of Chemistry and

7 Chemical Engineering, Jiangsu Key Laboratory of Vehicle Emissions Control,

$8 \quad$ Nanjing University, Nanjing 210023, China

$9{ }^{2}$ Green Catalysis Center, and College of Chemistry, Zhengzhou University,

10 Zhengzhou 450001, China

$11{ }^{3}$ Key Laboratory for Advanced Technology in Environmental Protection of Jiangsu

12 Province, Yancheng Institute of Technology, Yancheng, Jiangsu, 224051, China

13

14 


\section{Synthesis of Y-CDs and Y-CDs-TX.}

3 The Y-CDs were synthesized by solvothermal treatment of citric acid and

4 phenylenediamine, as shown in Figure S1. In a typical synthesis, citric acid (192 mg)

5 and diaminonaphthalene $(108 \mathrm{mg})$ were dissolved in Ultrapure water $(10 \mathrm{~mL})$. After

$630 \mathrm{~min}$ of sonication, the clear precursor solution was then transferred to a

7 poly(tetrafluoroethylene) (Teflon)-lined autoclave $(25 \mathrm{~mL})$ and heated at $160{ }^{\circ} \mathrm{C}$ for 8

$8 \mathrm{~h}$ in neutral environment. Citric acid produced the structural skeleton of the CDs

9 during the process of solvothermal treatment due to its low carbonization temperature.

10 O-phenylenediamine was used to introduce nitrogen into the structure of the CDs and

11 to passivate the trap states on the surfaces of the CDs with amino groups. After the

12 reaction, the solution was cooled to room temperature, either using water or air. The

13 Y-CDs were purified via centrifugation, filtration, and dialysis to remove the

14 byproducts and unreacted precursors, which could limit the intrinsic properties. The

15 resulting solution were purified via a centrifuge separates and dialysis $(\mathrm{MW}=1000)$.

16 After the process above, the Y-CDs-TX solution was made by mixing the Y-CDs (1

$17 \mathrm{mg} / \mathrm{mL})$ with an equal part of different concentration $\operatorname{TX}(0.1,0.05,0.01 \mathrm{M}$ etc.).

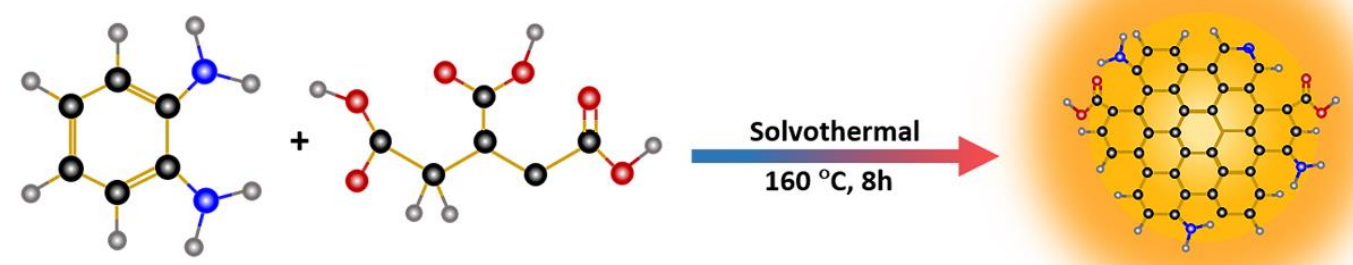

18

19 Figure S1 Schematic showing the synthetic strategy of Y-CDs. 
2 The spectral data, photostability, and the dynamics of the CDs were analyzed using a

3 home-built, widefield microscope. An excitation wavelength of $450 \mathrm{~nm}$ (super

4 continuous laser Fianium SC-400) and a power density of $48 \mathrm{~W} / \mathrm{cm}^{2}$ were applied on

5 the samples. The fluorescence of the CDs at different conditions were obtained using

6 an oil immersion objective lens (Olympus UPlanFLN $60 \times, \mathrm{NA}=1.25$ ) and detected

7 using an EMCCD camera (iXon Ultra 888, Andor), after being passed through a

8 470-nm longpass filter. For each field of view, time-stamped images were taken with

9 an exposure time of 100/500 ms per frame. A transmission grating (Newport, 150

10 lines per $\mathrm{mm}$ ) was placed in front of the camera for obtaining the fluorescence spectra.

11 PL lifetime measurements were done using a single-photon counting system (TCSPC,

12 Picoharp 300) with a 450-nm excitation light from a supercontinuous laser (Fianium

$13 \mathrm{SC}-400,40 \mathrm{MHz}$ ). For ensemble samples, the PL traces were extracted by averaging a

14 total of $100 \times 100$ pixels in the corresponding area. The net intensity was calculated by

15 subtracting the background for each frame. At least three replicates were measured for

16 each sample. The samples were performed under nitrogen atmosphere, unless

17 indicated. For single-particle CDs samples, the luminescence intensity of individual

18 CDs was calculated by averaging a total of $10 \times 10$ pixels for each dot and subtracting

19 the surrounding background. The single-particle trajectories were segmented to define

20 the "on" and "off" states, based on the change point analysis. The intensity threshold

21 was defined as the half of the segment with the lowest intensity and the highest

22 intensity. A segment of the trajectory whose intensity was above this threshold is 
1 defined as an "on" state and its corresponding duration is $\tau_{\mathrm{on}}$.

2 At least three replicates were measured for each sample. Most of the sample

3 preparations were performed under nitrogen atmosphere.

4 Glass Substrate Preparation.

5 High-quality coverslips from Corning, Inc. were used as the observation substrate. For

6 the ultrasonic treatment, the coverslips were cleaned in water followed by washing

7 alcohol for 40 min each. The coverslips were then rinsed in ultrapure water, dried with

8 nitrogen, and exposed to ultraviolet radiation for $1 \mathrm{~h}$ before use.

\section{Preparation of samples for single-particle measurement}

10 The samples were prepared by the ultrasonic dissolution of a suitable amount of the

11 CDs in appropriate solvents $(0.5 \mathrm{mg} / \mathrm{mL})$ and then diluting the solution to a

12 concentration of $5 \times 10^{-6}-5 \times 10^{-7} \mathrm{mg} / \mathrm{mL}$. Samples were prepared by the spin-coating

13 process. Individual particles were detected as bright diffraction-limited spots with the

14 same condition as ensemble sample.

15 Characterization of CDs

16 Transmission electron microscopy (TEM) images were acquired with a FEI

17 TECNAIG2F20-S-TWIN electron microscope. X-ray photoelectron spectroscopy

18 (XPS) measurements used a Thermo Fisher ESCALAB 250Xi surface analysis system.

19 X-ray diffraction (XRD) patterns were obtained using an X-ray diffractometer

20 (PANalytical, X'Pert PRO). The absorption and fluorescence spectra of the CDs were

21 recorded on a Persee TU-1810PC spectrophotometer and Edinburgh FLS 1000

22 fluorescence spectrophotometer at room temperature, respectively. Fourier transform 
1 infrared spectroscopy (FTIR) was performed on a Nexus 470 (Thermo Fisher)

2 spectrometer.

3 Fabrication and the character of LEDs

4 GaN LEDs chips without phosphor coating were purchased from Advanced

5 Optoeletronic Technology CO., Ltd. The emission peak of GaN LEDs chips located at

$6365 \mathrm{~nm}$ with the operating voltage of $4.0 \mathrm{~V}$. The composite of epoxy resin and CDs

7 (or CDs-TX) then used directly as a phosphor to drip onto the LEDs chip. The LEDs

8 chip was then placed in a $60^{\circ} \mathrm{C}$ oven for 2 hours. The emission spectra of LEDs were

9 measured by combining a Spectra scan PR-650 spectrophotometer with an integrating

10 sphere and a computer-controlled direct current power supply Keithley model 2400

11 voltage current source under ambient condition at room temperature. The color of the

12 light was identified by the CIE (Commission Internationale de L'Eclairage1931)

13 calorimeter system. All measurements were performed under dark condition.

14 Optical properties and chemical structure of Y-CDs

15 The PL emission peaks of Y-CDs did not shift when different excitation

16 wavelengths were applied. the time-resolved PL spectrum showed that a

17 double-exponential function could be fit over the entire emission spectra with a PL

18 lifetime of $2.52 \mathrm{~ns}$. The quantum yield of the Y-CDs was $7.44 \%$. 

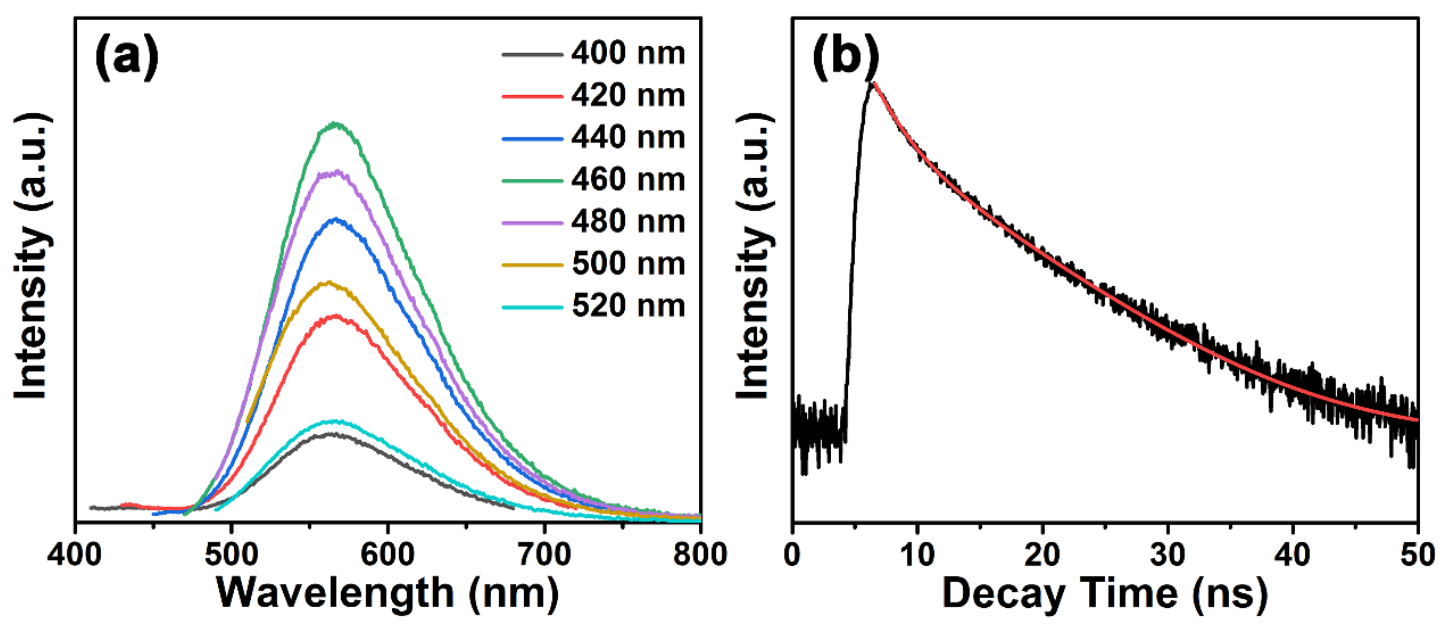

2 Figure S2 (a) Fluorescence emission spectra of Y-CDs in aqueous solution with

3 progressively longer excitation wavelengths from $400 \mathrm{~nm}$ to $520 \mathrm{~nm}$ (b)

4 Photoluminescence decay of CDs aqueous solution under $450 \mathrm{~nm}$ excitation.

The chemical structure of the Y-CDs was characterized by X-ray photoelectron

6 spectroscopy (XPS) and Fourier-transform infrared spectroscopy (FTIR).The N 1s

7 band also had three component peaks at 399.0, 400.2, and $401.1 \mathrm{eV}$, corresponding to

8 pyridinic $\mathrm{N}$, pyrrolic $\mathrm{N}$, and graphitic $\mathrm{N}$, respectively (Figure $\mathrm{S} 3 \mathrm{a}$ ). The two $\mathrm{O} 1 \mathrm{~s}$

9 peaks at 531.5 and $532.5 \mathrm{eV}$ arose from $\mathrm{C}=\mathrm{O}$ and $\mathrm{C}-\mathrm{O},{ }^{1}$ respectively (Figure $\mathrm{S} 3 \mathrm{~b}$ ). It

10 can be concluded that the percentages of $\mathrm{C}, \mathrm{N}$, and $\mathrm{O}$ in $\mathrm{Y}$-CDs are $77.2 \%, 15.2 \%$,

11 and $7.6 \%$, respectively, while the percentages of $\mathrm{C}, \mathrm{N}$, and $\mathrm{O}$ in $\mathrm{Y}$-CDs-TX are $80.2 \%$,

$128.2 \%$ and $11.6 \%$, respectively. The decrease in the proportion of $\mathrm{N}$ in Y-CDs-TX is

13 attributed to Trolox addition. The FTIR spectrum of the Y-CDs showed bands at

$14 \quad 1500-1620$ and $920-850 \mathrm{~cm}^{-1}$, indicating the existence $\mathrm{C}=\mathrm{C}$ and $\mathrm{C}=\mathrm{N}$ of conjugated

15 aromatic structures (Figure S4). The bands at 1160 and $1270 \mathrm{~cm}^{-1}$ corresponded to the

16 stretching vibrations of $\mathrm{C}-\mathrm{O}$ and $\mathrm{C}-\mathrm{N}$, respectively. The bands from 3300 to 3500

$17 \mathrm{~cm}^{-1}$ were attributed to the stretching vibrations of $\mathrm{O}-\mathrm{H}$ and $\mathrm{N}-\mathrm{H},{ }^{2-3}$ respectively. The 
1 FTIR and XPS analyses above revealed that abundant hydrophilic functional groups

2 not only provided interaction sites for specific compounds but improved the dispersity.

3 Transmission electron microscopy (TEM) images revealed that the Y-CDs were

4 nanodots with an average lateral size of $4.6 \mathrm{~nm}$ (Figure S5a, b). High-resolution TEM

5 showed Y-CDs with a lattice spacing of $0.21 \mathrm{~nm}$, consistent with the (100) lattice

6 spacing of graphene (inset of Figure S5c). X-ray powder diffraction of the Y-CDs

7 showed a broad peak centered at about $25^{\circ}$ (Figure S5d). Both the X-ray diffraction

8 (XRD) and TEM data indicated that high-quality Y-CDs had been successfully

9 prepared.

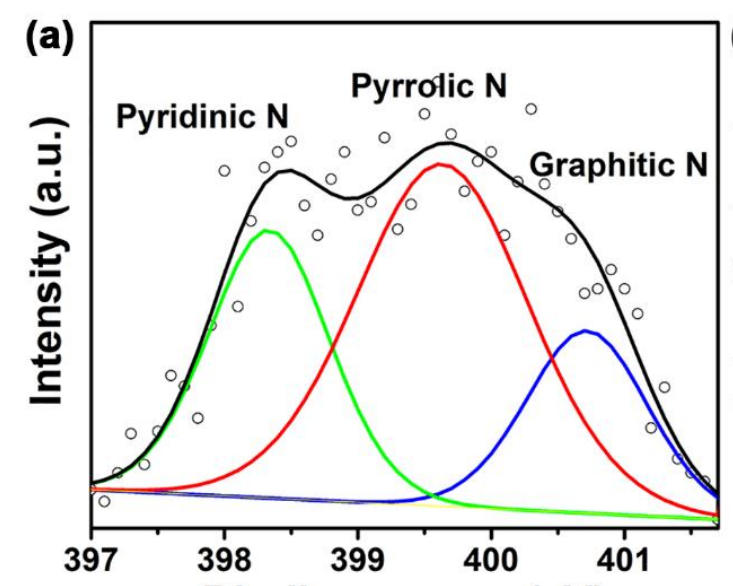

10

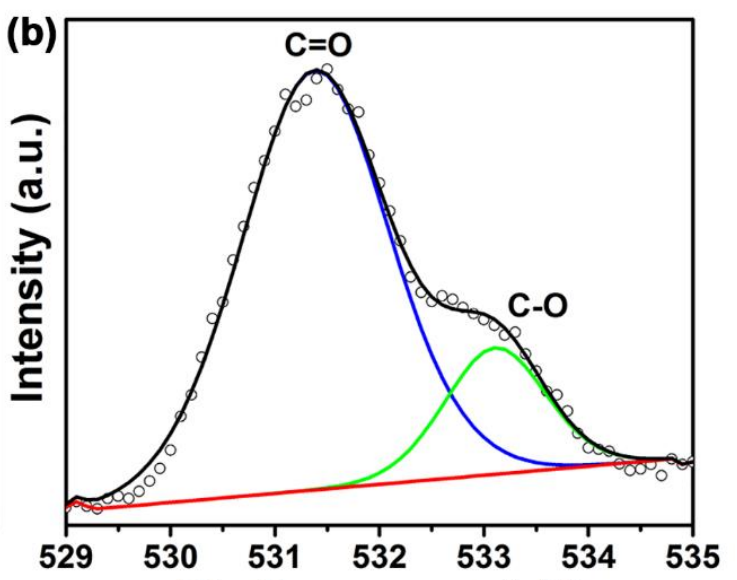

Binding energy (eV)

11 Figure S3 XPS spectrum of Y-CDs (a) N1s spectrum, (b) O1s spectrum. 


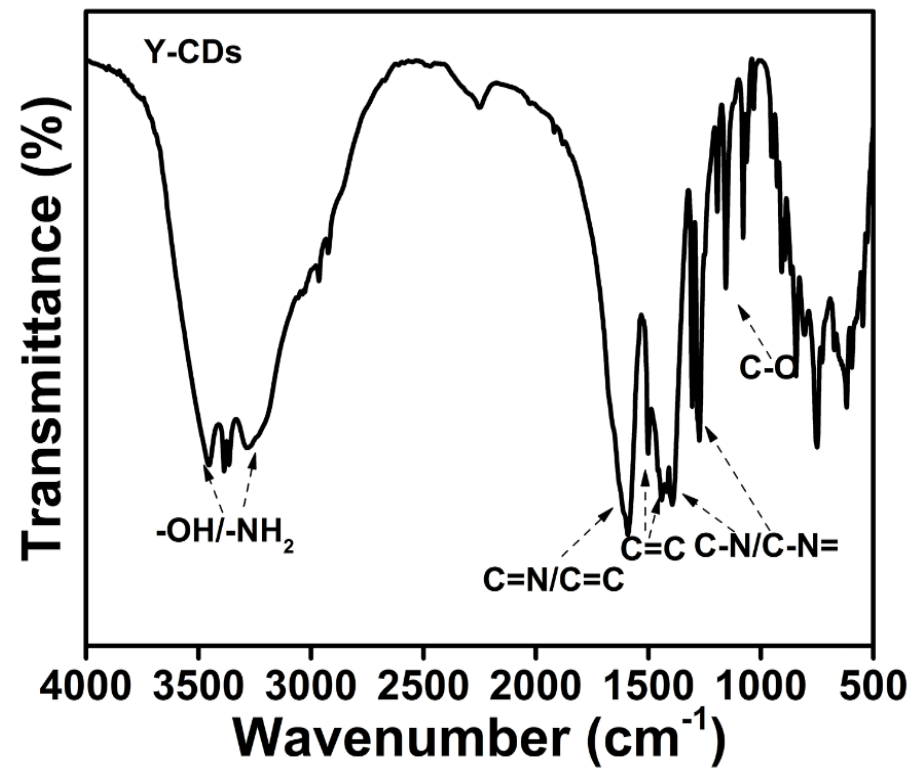

2 Figure S4 FTIR spectrum of Y-CDs.
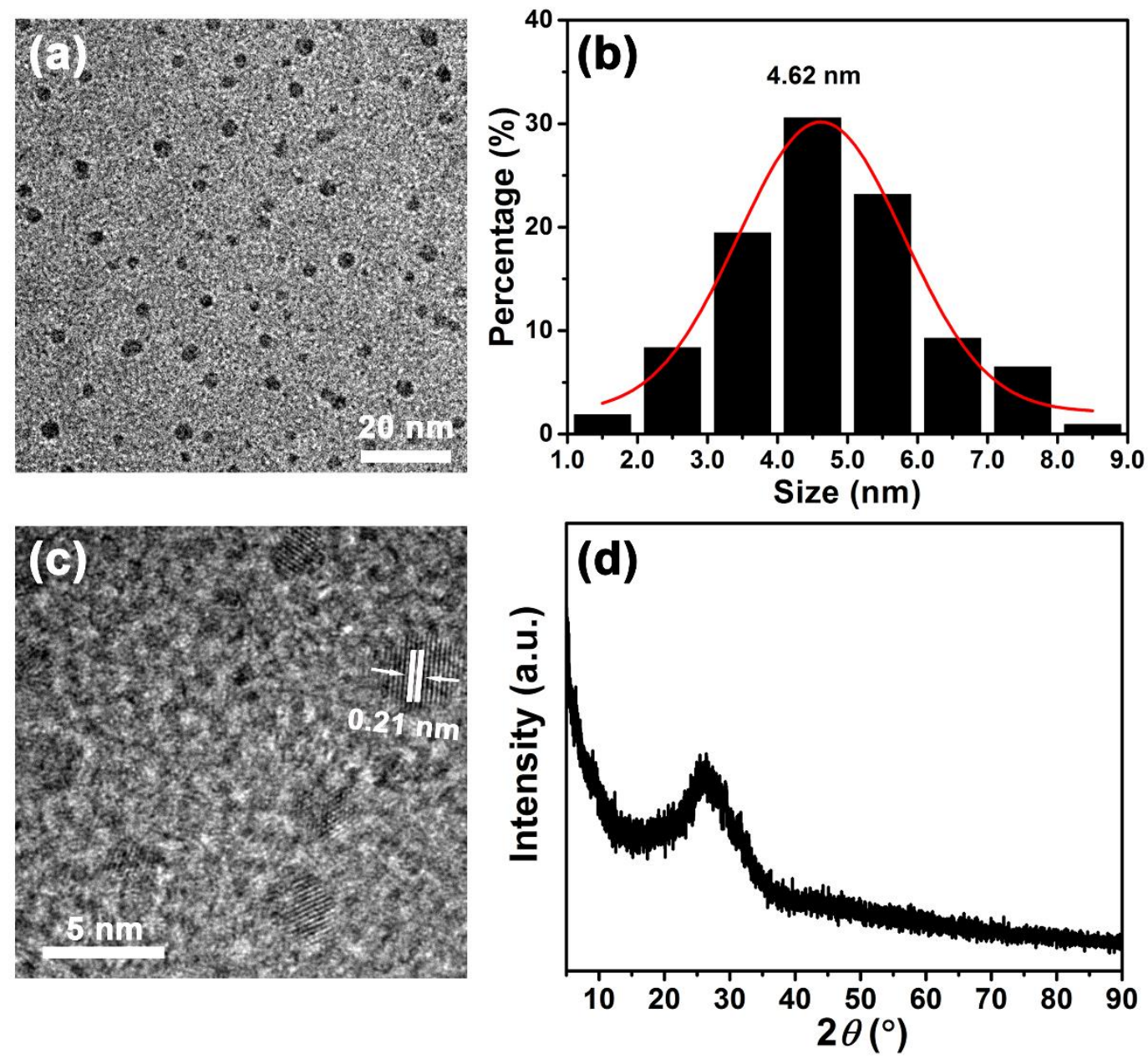

4 Figure S5 Structural characterizations of Y-CDs (a) TEM image, (b) corresponding

5 size distributions of Y-CDs, (c) High-resolution TEM and (d) XRD of Y-CDs. 

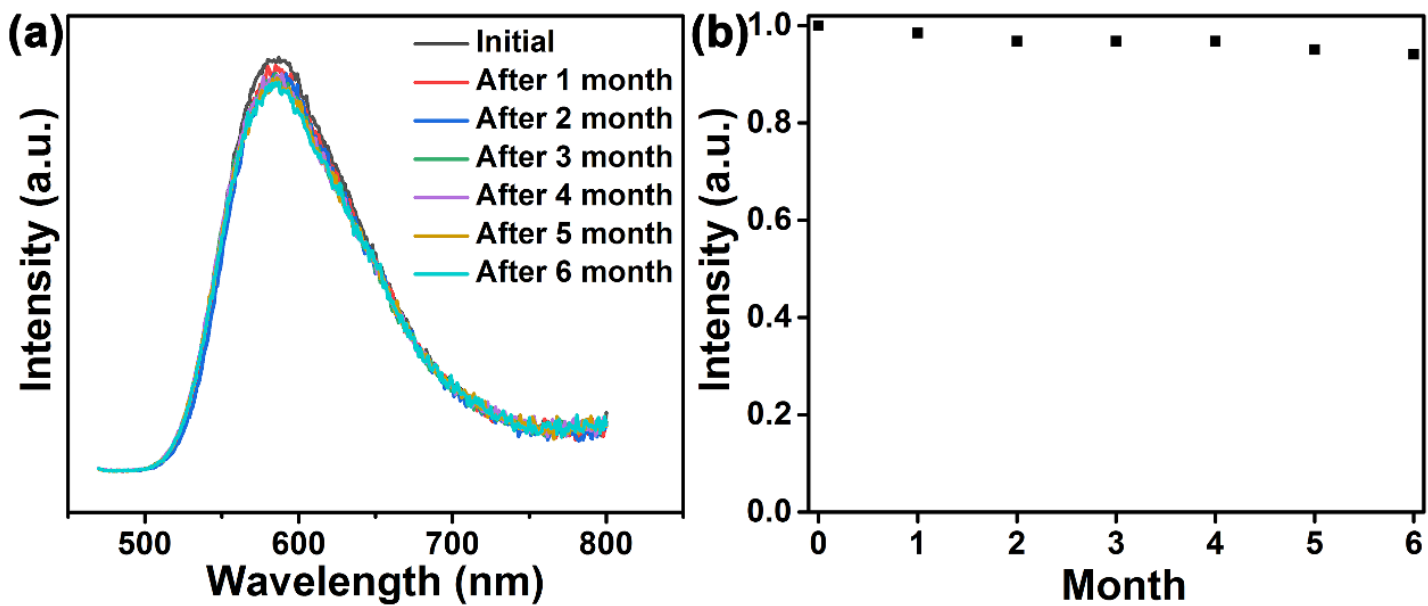

2 Figure S6 The stability test of fluorescence intensity on storage time for Y-CDs in DI

3 water.

5 Figure S7 The photoluminescence enhancement curves of fixing the concentration of

$6 \quad$ Y-CDs and adjusting only the concentration of TX.

8 two spectra are very similar, showing $\mathrm{O}-\mathrm{H}$ and $\mathrm{N}-\mathrm{H}$ at $3300-3500 \mathrm{~cm}^{-1}, \mathrm{C}=\mathrm{C}$ and

$9 \mathrm{C}=\mathrm{N}$ at $1500-1620 \mathrm{~cm}^{-1}, \mathrm{C}-\mathrm{N} / \mathrm{C}-\mathrm{N}=$ at $1270-1400 \mathrm{~cm}^{-1}$ and $\mathrm{C}-\mathrm{O}$ at $1157 \mathrm{~cm}^{-1}$. The

10 extra peak at $1720 \mathrm{~cm}^{-1}$ of Y-CDs-TX is attributed to the stretching vibration of $\mathrm{C}=\mathrm{O}$

11 from Trolox. The FTIR analysis results suggest that no chemical bonding between

12 Y-CDs and Trolox which was further proved by XPS analysis. The results of XPS 
1 measurements of Y-CDs and Y-CDs-TX are also remained unchanged as shown in

2 Fig.1 c, Fig.S3, Fig. S8, Table R1, respectively. In general, it is difficult for amino and

3 carboxyl groups to react without an activator at room temperature (common amino

4 activators such as NHS, common carboxyl activators such as EDC). Therefore, it is

5 reasonable to believe the Y-CDs and TX would not form covalent bonds.
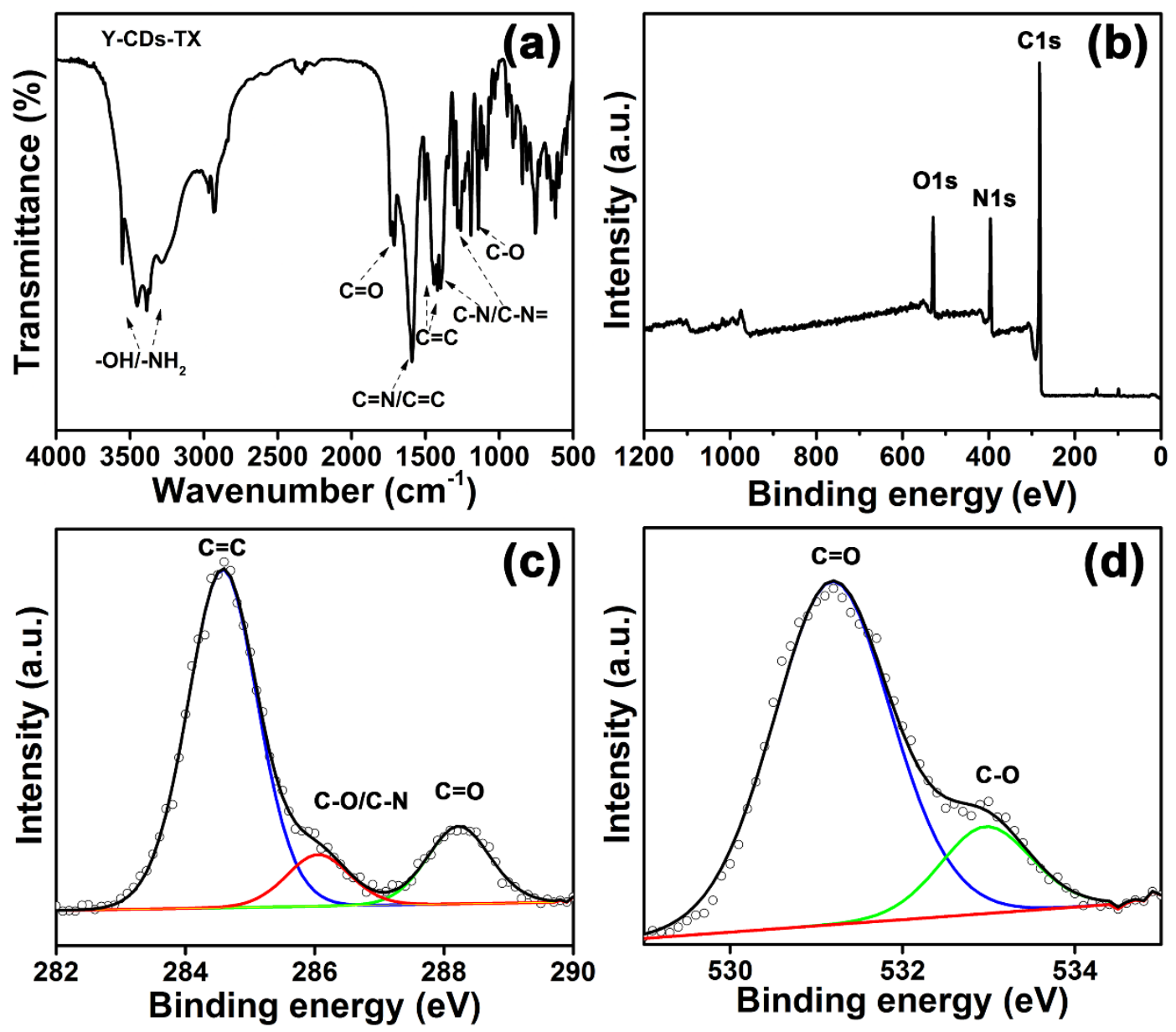

6

$7 \quad$ Figure S8 (a) FTIR spectrum of Y-CDs-TX, (b) XPS survey spectra, (c) C1s XPS

8 spectrum and (d) O1s spectrum of Y-CDs-TX. 

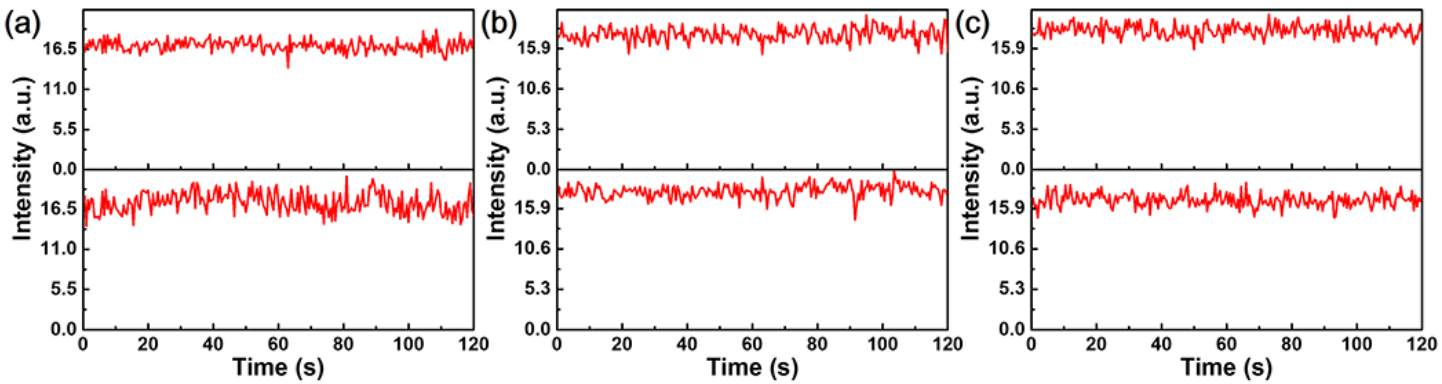

2 Figure S9 Typical PL intensity traces of individual Y-CD-TX.
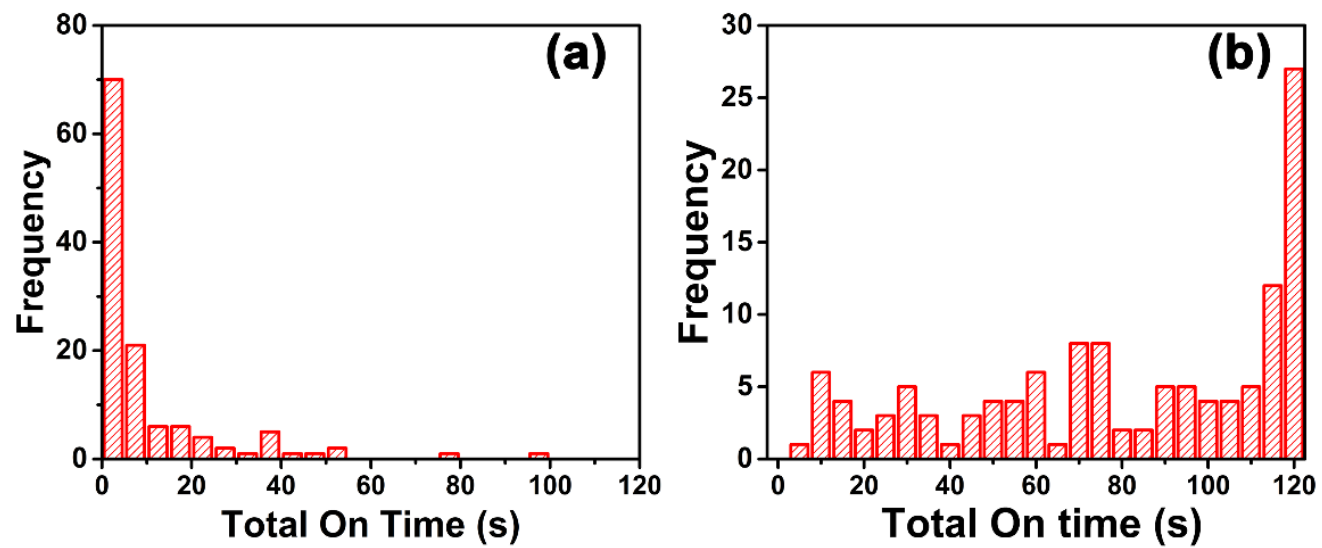

5 Figure S10 (a) The total "on" state dwell time of Y-CDs (a) and Y-CDs-TX (b).

6 Table S1 Content of Various Chemical Bonds in Y-CDs and Y-CDs-TX

\begin{tabular}{ccccccc}
\hline & \multicolumn{3}{c}{$\mathrm{C} 1 \mathrm{~s}$} & & \multicolumn{2}{c}{$\mathrm{O} 1 \mathrm{~s}$} \\
\cline { 2 - 4 } \cline { 6 - 7 } \cline { 5 - 7 } samples & $\mathrm{C}=\mathrm{C}$ & $\mathrm{C}-\mathrm{O} / \mathrm{C}-\mathrm{N}$ & $\mathrm{C}=\mathrm{O}$ & & $\mathrm{C}=\mathrm{O}$ & $\mathrm{C}-\mathrm{O}$ \\
$\mathrm{Y}-\mathrm{CDs}$ & $74.16 \%$ & $10.25 \%$ & $15.59 \%$ & & $81.68 \%$ & $18.32 \%$ \\
$\mathrm{Y}-\mathrm{CDs}-\mathrm{T}$ & $74.10 \%$ & $10.32 \%$ & $15.58 \%$ & & $81.64 \%$ & $18.36 \%$ \\
$\mathrm{X}$ & & & & & & \\
\hline
\end{tabular}

\section{References}

10 (1) Lu, S.; Sui, L.; Liu, J.; Zhu, S.; Chen, A.; Jin, M.; Yang, B. Near-Infrared

11 Photoluminescent Polymer-Carbon Nanodots with Two-Photon Fluorescence. Adv.

12 Mater. 2017, 29, 1603443. 
1 (2) Vallan, L.; Urriolabeitia, E. P.; Ruipérez, F.; Matxain, J. M.; Canton-Vitoria, R.;

2 Tagmatarchis, N.; Benito, A. M.; Maser, W. K. Supramolecular-Enhanced Charge 3 Transfer within Entangled Polyamide Chains as the Origin of the Universal Blue 4 Fluorescence of Polymer Carbon Dots. J. Am. Chem. Soc. 2018, 140, 12862-12869.

5 (3) Wang, L.; Zhu, S.; Wang, H.; Qu, S.; Zhang, Y.; Zhang, J.; Chen, Q.; Xu, H.; 6 Han, W.; Yang, B.et al. Common Origin of Green Luminescence in Carbon Nanodots 7 and Graphene Quantum Dots. Acs Nano 2014, 8, 2541-2547.

8

9 